\title{
Prenatal Tobacco Exposure and Brain Morphology: A Prospective Study in Young Children
}

\author{
Hanan El Marroun*, 1,2 , Marcus N Schmidt ${ }^{1,2}$, Ingmar HA Franken ${ }^{1,3}$, Vincent WV Jaddoe ${ }^{2,4,5}$, \\ Albert Hofman ${ }^{5}$, Aad van der Lugt ${ }^{6}$, Frank C Verhulst ${ }^{1}$, Henning Tiemeier ${ }^{1,5,7}$ and Tonya White ${ }^{1,6}$ \\ 'The Department of Child and Adolescent Psychiatry, Erasmus MC, Sophia Children's Hospital, Rotterdam, The Netherlands; ${ }^{2}$ The Generation R \\ Study Group, Erasmus MC, Rotterdam, The Netherlands; ${ }^{3}$ Institute of Psychology, Erasmus University Rotterdam, Rotterdam, The Netherlands; \\ ${ }^{4}$ The Department of Pediatrics, Erasmus MC, Rotterdam, The Netherlands; ${ }^{5}$ The Department of Epidemiology, Erasmus MC, Rotterdam, \\ The Netherlands; ${ }^{6}$ The Department of Radiology, Erasmus MC, Rotterdam, The Netherlands; ${ }^{7}$ The Department of Psychiatry, Erasmus MC, \\ Rotterdam, The Netherlands
}

It is well known that smoking during pregnancy can affect offspring health. Prenatal tobacco exposure has been associated with negative behavioral and cognitive outcomes in childhood, adolescence, and young adulthood. These associations between prenatal tobacco exposure and psychopathology in offspring could possibly be explained by the influence of prenatal tobacco exposure on brain development. In this prospective study, we investigated the association between prenatal tobacco exposure, behavioral and emotional functioning and brain morphology in young children. On the basis of age and gender, we matched II 3 children prenatally exposed to tobacco with 113 unexposed controls. These children were part of a population-based study in the Netherlands, the Generation R Study, and were followed from pregnancy onward. Behavioral and emotional functioning was assessed at age 6 with the Child Behavior Checklist. We assessed brain morphology using magnetic resonance imaging techniques in children aged 6-8 years. Children exposed to tobacco throughout pregnancy have smaller total brain volumes and smaller cortical gray matter volumes. Continued prenatal tobacco exposure was associated with cortical thinning, primarily in the superior frontal, superior parietal, and precentral cortices. These children also demonstrated increased scores of affective problems. In addition, thickness of the precentral and superior frontal cortices was associated with affective problems. Importantly, brain development in offspring of mothers who quit smoking during pregnancy resembled that of nonexposed controls (no smaller brain volumes and no thinning of the cortex). Our findings suggest an association between continued prenatal tobacco exposure and brain structure and function in school-aged children.

Neuropsychopharmacology (2014) 39, 792-800; doi:I0.1038/npp.2013.273; published online 30 October 2013

Keywords: prenatal tobacco exposure; pediatric brain development; brain morphology

\section{INTRODUCTION}

It is well known that cigarette smoking can cause serious health problems, including cardiovascular diseases and cancer (Steliga and Dresler, 2011). Smoking during pregnancy has also been shown to adversely affect offspring health (Rogers, 2009). Yet, up to $25 \%$ of pregnant women report smoking during pregnancy (Cnattingius, 2004). Smoking during pregnancy has been linked to spontaneous abortions (Nielsen et al, 2006), reduced intrauterine growth (Abbott and Winzer-Serhan, 2012), and sudden infant death syndrome (DiFranza and Lew, 1995). During childhood and adolescence, prenatal tobacco exposure has been associated with behavioral and cognitive problems (Huizink and Mulder,

*Correspondence: Dr H El Marroun, The Department of Child and Adolescent Psychiatry, Erasmus MC, Sophia Children's Hospital, PO Box 2060, Rotterdam 3000 CB, The Netherlands, Tel: +3I 10-7034275, Fax: +31 10-7044645, E-mail: h.marrounel@erasmusmc.nl

Received 30 May 2013; revised I August 2013; accepted 20 September 2013; accepted article preview online 7 October 2013
2006). Further, evidence is accumulating that prenatal tobacco exposure is related to psychiatric disorders and mortality from childhood to young adulthood (Weissman et al, 1999; Ekblad et al, 2010). Thus, having a better understanding of how alterations in the brain due to prenatal exposure to cigarettes contribute to at-risk states in children is important.

Although little is known about how prenatal tobacco exposure influences human brain development, evidence from animal studies show nicotine-induced changes in their neurotransmitter systems (Slotkin, 2004). These changes may consequently have adverse effects on specific neurodevelopmental processes. Roy et al (1998) observed a reduction in brain weight, cortical thickness, and neural density after prenatal nicotine exposure in rats (Roy and Sabherwal, 1994). Similar differences in the fetal and neonatal period have been shown in offspring of women who smoke during pregnancy, including reduced fetal head growth, reduced cerebellar growth, and smaller width of the lateral ventricles (Roza et al, 2007). Moreover, smaller frontal lobe and cerebellar volumes (Ekblad et al, 2010) have been reported in tobacco-exposed neonates. Further, 
there is evidence that these alterations in the neonatal period persist into childhood and adolescence. Exposure to cigarette smoking has been associated with reduced cerebral cortical gray matter volume in children (Rivkin et al, 2008), and smaller subcortical gray matter volumes, including the amygdala, thalamus, and pallidum (Haghighi et al, 2013; Liu et al, 2013). In addition, prenatal tobacco-exposed adolescents have a reduced corpus callosum volume (Paus et al, 2008) and thinner orbitofrontal, middle frontal, and parahippocampal cortices (Toro et al, 2008; Lotfipour et al, 2009). However, a potential confounder is that these adolescents may have smoked themselves, as prenatally exposed children are more vulnerable to use tobacco themselves (Cornelius et al, 2000; Buka et al, 2003). Thus, it cannot be completely ruled out that these differences were a result of smoking during adolescence.

The current study examined a nonclinical population-based group of children aged 6-8 years, a time before risk period of smoking. The aim of this study was to investigate whether prenatal exposure to tobacco is related to brain morphology in childhood. In addition, we aimed to investigate whether smoking during pregnancy is associated with behavioral and emotional problems and whether these problems are mediated by differences in brain morphology. On the basis of prior animal and human research that smoking during pregnancy results in decreased brain growth as well as cortical, subcortical, and cerebellar differences, we hypothesized that prenatal exposure to tobacco would be associated with more widespread abnormalities in the brain together with behavioral and/or emotional problems in childhood.

\section{MATERIALS AND METHODS}

\section{Participants}

This study reports findings from an ongoing populationbased prospective cohort in Rotterdam (the Netherlands), the Generation $\mathrm{R}$ Study. The design has been described previously (Jaddoe et al, 2010) and was approved by the Medical Ethics Committee of the Erasmus Medical Center. Written informed consent was obtained from all participants.

In September 2009, 6- to 8-year-old children from the Generation R Study were invited to participate in a magnetic resonance imaging (MRI) component of the study. Detailed information can be found in White et al (2013). Approximately $20 \%$ declined to participate. Exclusion criteria were the following: having a significant motor or sensory disorder, moderate-to-severe head trauma with loss of consciousness, neurological disorders, claustrophobia, and contraindications to MRI (eg, having a pacemaker).

For this study, we matched children prenatally exposed to tobacco $(n=113)$ with controls based on their age and gender using a 'fuzzy' matching procedure. This procedure randomly searches for a case-control match that falls within the set of defined criteria: an exact match for gender and a fuzzy match for age with a difference of 4 months (smaller age differences did not yield a match for each exposed child).

\section{Tobacco Exposure During Pregnancy}

Information about maternal smoking was prospectively obtained by postal questionnaires in each trimester (Roza et al, 2007). Maternal smoking at enrollment was assessed in the first questionnaire by asking whether the mother smoked during pregnancy. In the second and third questionnaires (mid- and late pregnancy), mothers were asked whether they had smoked in the last 2 months. Maternal smoking during pregnancy was categorized on the basis of all three questionnaires into 'no smoking during pregnancy', 'until pregnancy was known', and 'continued during pregnancy'. Children of mothers who reported in the first questionnaire to have smoked until pregnancy was known, but reported in the second or third questionnaire to have smoked during pregnancy were classified as 'continued during pregnancy' group. Similarly, children of mothers who reported no smoking in the first but acknowledged to smoke in the second or third questionnaire were classified as 'continued during pregnancy'. When information was missing on maternal smoking at enrollment, information from the second and/or third questionnaire was used to classify the subjects into 'no smoking in pregnancy' or 'continued smoking in pregnancy'.

The number of cigarettes smoked per day was compiled into three categories 'less than 5 cigarettes per day', 'between 5 cigarettes per day', and 'more than 9 cigarettes per day' (only eight pregnant mothers smoked more than 20 cigarettes per day in one of the trimesters). At the time of scanning, all researchers were blind to exposure status.

\section{Magnetic Resonance Imaging}

Children were familiarized with the MRI environment during a mock session. All images were acquired using the same sequence on the same scanner, located at the Department of Radiology in the Erasmus Medical Center. Images were acquired on a 3Tesla scanner (750 Discovery, GE Healthcare, Milwaukee, WI) using an eight-channel head coil. Care was taken so that children were comfortable in the scanner and soft cushions were used for head immobilization. Following a three-plane localizing scan, a highresolution $\mathrm{T} 1$ inversion recovery fast-spoiled gradient recalled sequence was acquired in the sagittal plane with the following parameters: $\mathrm{TE}=4.24 \mathrm{~ms}, \mathrm{TI}=350 \mathrm{~ms}$, $\mathrm{TR}=10.26 \mathrm{~ms}, \mathrm{NEX}=1$, flip angle $=16^{\circ}$, and resolution $0.9 \mathrm{~mm}^{3}$ isotropic. At the scanner, structural images were rated for quality on a five-level Likert scale: unusable, poor (but useable), sufficient, very good, and excellent. If the initial T1 scan was rated as poor, the T1 sequence was repeated, and the better of the two scans was used.

\section{Cortical and Volumetric Measures and Cortical Thickness}

Cortical reconstruction and volumetric segmentation was performed with the Freesurfer image analysis suite version 5.1.0 (http://surfer.nmr.mgh.harvard.edu/). Freesurfer computes these measures in an automated approach, and technical procedures have been described extensively (Fischl and Dale, 2000; Fischl et al, 2002; Fischl et al, 2004). Cortical thickness measurements have been validated against histological analysis (Rosas et al, 2002) and manual measurements (Kuperberg et al, 2003; Salat et al, 2004). Freesurfer morphometry has demonstrated good test-retest reliability across scanner manufacturers and field strengths 
(Han et al, 2006; Reuter et al, 2012). Numerous studies using Freesurfer in typical and atypical developing schoolaged children are available (O'Donnell et al, 2005; Derauf et al, 2009; Ghosh et al, 2010; Ducharme et al, 2012). All Freesurfer output was visually inspected for quality control. There were no problems with registration and skull stripping, but in some cases segmentation was suboptimal because of movement $(n=19)$.

\section{Child Behavioral and Emotional Problems}

We assessed child behavioral and emotional problems using the Child Behavior Checklist for toddlers (CBCL/1 $1 / 2-5)$, which is a standardized instrument and covers a broad age range. Mothers filled out the $\mathrm{CBCL} / 1^{1 / 2-5}$ at 6 years of age (as a large proportion of children were younger than 6 years). The Dutch version of the CBCL/1 $1 / 2-5$ is reliable and well validated and the subscales for syndromes derived from the $\mathrm{CBCL} / 1 \frac{1 / 2-5}{2}$ have been consistent across 23 international studies involving diverse societies (Ivanova et al, 2010). The five scales (Affective Problems, Anxiety Problems, Pervasive Developmental Problems, Attention-Deficit/Hyperactivity Problems, and Oppositional Defiant Problems) can be derived from the CBCL $/ 1 \frac{1 / 2-5}{2}$ are consistent with the Diagnostic and Statistical Manual of Mental Disorders-4th edition diagnostic categories.

\section{Additional Measures}

Maternal characteristics were the following: maternal age at intake, educational level, ethnicity, and drinking habits. Child characteristics were the following: age at MRI assessment, gender, nonverbal IQ at 6 years, gestational age at birth, and birth weight. Maternal educational level was categorized into three levels: primary (no or primary education), secondary (lower and intermediate vocational training), and higher (higher vocational education and university) education. Maternal ethnicity was defined according to the classification of Statistics Netherlands; a mother was classified as being non-Dutch if one of her parents was born abroad. If both of her parents were born abroad, the country of birth of the mother's mother determined maternal ethnicity. Information on maternal drinking habits, like information on maternal smoking habits, was collected with questionnaires in each trimester. Nonverbal IQ was assessed at the age of 6 years with two subtests of a revised Dutch intelligence test, the 'SnijdersOomen Niet-verbale intelligentie Test-Revisie (SON-R 2.5-7)': Mosaics and Categories(Tellegen et al, 2005). Gestational age (in weeks) and weight at birth (in grams) were extracted from medical records.

\section{Statistical Analyses}

Demographic measures and volumetric measures. Differences in demographics, cognitive, and clinical variables between the tobacco-exposed (quit and continued smoking) and nonexposed groups were determined using $\chi^{2}$-tests for categorical data and (paired) $t$-tests for continuous variables. Volumetric measures from the Freesurfer segmentation were used to compare brain morphology. If global differences in total brain volume were present, post hoc analyses were performed to assess which global (cortical volume, cortical gray matter volume, cortical white matter volume, and ventricular volume) were involved. These tests were performed using paired linear regression analyses. Further, we tested whether there was a dose-response relationship between prenatal tobacco exposure ('less than five cigarettes per day', 'between five and nine cigarettes per day', and 'more than nine cigarettes per day) and brain morphology using this information as a continuous (ie, ordinal) and categorical variable.

We also investigated whether subcortical volumes, ie, caudate, putamen, pallidum, accumbens, hippocampus, amygdala, and thalamus differed between the groups. Supplemental analyses were performed that took into account total brain volume.

We investigated whether maternal smoking was associated with child behavioral and emotional problems using paired regression analyses. We then included brain measures as a variable to explore whether the association was explained by these brain measures. In addition, we also performed separate regression analyses to investigate the association between brain measures and child behavioral and emotional problems.

In all models, subjects were matched on age and gender, and we adjusted for maternal education, ethnicity, alcohol use during pregnancy, and birth weight. Covariates were selected based on prior literature or the $5 \%$ changein-estimate criterion. These analyses were performed in the Statistical Package for the Social Sciences (SPSS) Version 20.0.

Surface group analyses. In addition to hypothesis-driven analyses investigating global differences, we also performed data-driven analyses to assess whether regional differences exist. Differences in cortical thickness and surface area (outcomes), smoothed with a 10-mm full-width halfmaximum Gaussian kernel, for both hemispheres between tobacco-exposed and unexposed subjects (predictor) were analyzed using paired analysis within Freesurfer with the built-in general linear model module (mri_glmfit). This datadriven procedure allows for generation of statistical parametric maps that can be thresholded; we used an uncorrected threshold of $p<0.05$ for initial vertexwise comparison. Thereafter, a clusterwise correction for multiple comparisons was performed using the built-in simulation procedure with 5000 iterations and a clusterwise threshold of $p<0.05$, which controls for the rate of false-positive clusters (Hagler et al, 2006). We used these surface analyses as a guide to regions of interest. Post hoc, we extracted these clusters (cluster size $>400 \mathrm{~mm}^{2}$ in both hemispheres) as an outcome into paired multiple linear regression analysis in order to determine whether differences in these clusters were confounded by any of the covariates. As there was no specific hypothesis on differential effects between the two hemispheres, we calculated a weighted average cortical thickness of the regions of interest (weighted as surface areas could differ in size). We performed the same analysis with a 5- and 15-mm Gaussian kernel, and the results were similar (data not shown). 
Analyses were performed using all subjects in which Freesurfer data were available, irrespective of image quality. We then performed the same analyses, excluding those subjects with poor quality of the data $(n=12)$ and subjects with suboptimal Freesurfer registration $(n=19)$. As we performed paired analyses, 31 pairs were excluded. The results remained very similar after excluding these data, and therefore we only reported the results of the analysis including all subjects $(n=226)$.

\section{RESULTS}

\section{Demographic Measures and Volumetric Measures}

Demographic characteristics for this sample are reported in Table 1. The sample was categorized in three groups: the nonexposed controls, children of whom the mother smoked until pregnancy was known (early pregnancy exposure), and children of whom the mother continued to smoke during pregnancy (multiple trimester exposure). Compared with nonexposed controls, children exposed to continued tobacco smoking were more likely to have a lower weight at birth (3194 $\pm 536 v s 3475 \pm 520 \mathrm{~g}, p=0.001$ ). No differences between the groups were found on other variables including maternal age, educational level, ethnicity, drinking habits, child gender and age, and nonverbal IQ. Overall, the MRI image quality was good and did not differ between the groups (Table 1).

Table 2 demonstrates an association between continued maternal smoking and smaller total brain volume $(B=-55.3,95 \%$ CI: -93.5 to $-17.0, p=0.005)$; this association did not change after adjustment for the covariates. Subsequently, this table shows that continued maternal smoking was associated with smaller cortical gray $(B=-39.3,95 \%$ CI: -66.0 to $-12.6, p=0.004)$ and white matter volumes $(B=-16.3,95 \% \mathrm{CI}$ : -30.4 to -2.2 , $p=0.02$ ); again, these associations could not be explained by the included covariates. Interestingly, maternal smoking in early pregnancy only was not associated with differences in global brain volumes (Table 2).

Continued maternal smoking was associated with a smaller caudate nucleus $(B=-482.0,95 \% \mathrm{CI}$ : -819.3 to -144.8 , $p=0.003)$ and a smaller accumbens area $(B=-90.6,95 \%$ CI: 164.6 to $-16.6, p=0.02$ ), but was not associated with the other subcortical structures. We then constructed total brain volume-adjusted volumes (ie, volume/total brain volume). No association between continued maternal smoking and total brain volume-adjusted caudate volume $(B=-0.07$, $95 \% \mathrm{CI}:-0.41$ to $0.26, p=0.68)$, or total brain volumeadjusted accumbens $(B=-0.01,95 \%$ CI: -0.09 to 0.06 , $p=0.73$ ) was observed. No relation was seen between maternal smoking in early pregnancy and the volumes of the subcortical structures.

\section{Cortical Surface Analyses}

Figure 1 shows that tobacco-exposed children (continued maternal smoking) had thinner cortices in several brain regions. These thinner cortices were more pronounced in the left hemisphere, but thinner cortices were also seen in similar locations in the right hemisphere. Left hemisphere clusters larger than $400 \mathrm{~mm}^{2}$ were the following: the superior frontal, superior parietal, inferior temporal,
Table I Descriptive Statistics of Whole-Study Population

\begin{tabular}{|c|c|c|c|}
\hline & $\begin{array}{l}\text { Nonexposed } \\
\text { controls }\end{array}$ & $\begin{array}{l}\text { Quit smoking } \\
\text { when } \\
\text { pregnancy } \\
\text { was known }\end{array}$ & $\begin{array}{l}\text { Continued } \\
\text { smoking } \\
\text { (multiple } \\
\text { trimesters) }\end{array}$ \\
\hline & $\mathbf{N}=I / 3$ & $\mathbf{N}=17$ & $\mathbf{N}=96$ \\
\hline \multicolumn{4}{|l|}{ Maternal characteristics } \\
\hline Maternal age at intake & $30.5 \pm 4.5$ & $29.8 \pm 5.6$ & $29.3 \pm 5.8$ \\
\hline \multicolumn{4}{|l|}{ Educational level (\%) } \\
\hline Primary & 8.0 & 5.9 & 10.4 \\
\hline Secondary & 46.0 & 52.9 & 66.6 \\
\hline Higher & 46.0 & 41.2 & 23.0 \\
\hline \multicolumn{4}{|l|}{ Ethnicity } \\
\hline Dutch & 67.3 & 58.8 & 53.1 \\
\hline Non-Dutch Western & 5.3 & 5.9 & 8.3 \\
\hline $\begin{array}{l}\text { Non-Dutch Non- } \\
\text { Western }\end{array}$ & 27.4 & 35.3 & 38.6 \\
\hline \multicolumn{4}{|l|}{ Drinking habits (\%) } \\
\hline Never drank in pregnancy & 41.6 & 23.5 & 30.2 \\
\hline $\begin{array}{l}\text { Drank until pregnancy } \\
\text { was known }\end{array}$ & 12.4 & 17.6 & 12.5 \\
\hline $\begin{array}{l}\text { Continued to drink in } \\
\text { pregnancy }\end{array}$ & 46.0 & 58.8 & 57.3 \\
\hline \multicolumn{4}{|l|}{ Smoking habits (\%) } \\
\hline $\begin{array}{l}\text { Never smoked in } \\
\text { pregnancy }\end{array}$ & 100.0 & - & - \\
\hline $\begin{array}{l}\text { Less than five cigarettes } \\
\text { per day }\end{array}$ & - & 52.9 & 29.2 \\
\hline $\begin{array}{l}\text { Between five and nine } \\
\text { cigarettes per day }\end{array}$ & - & 23.5 & 17.7 \\
\hline $\begin{array}{l}\text { More than nine cigarettes/ } \\
\text { day }\end{array}$ & - & 23.5 & 53.1 \\
\hline \multicolumn{4}{|l|}{ Child characteristics } \\
\hline Age at MRI (months) & $87.2 \pm 9.2$ & $89.4 \pm 10.4$ & $86.2 \pm 8.9$ \\
\hline Gender (\% boys) & 58.4 & 58.8 & 58.3 \\
\hline $\begin{array}{l}\text { Nonverbal IQ at age } \\
6 \text { years }\end{array}$ & $101.0 \pm 12.8$ & $103.6 \pm 14.4$ & $99.6 \pm 12.7$ \\
\hline $\begin{array}{l}\text { Gestational age at birth } \\
\text { (weeks) }\end{array}$ & $40.0 \pm 1.6$ & $39.2 \pm 2.2^{\mathrm{a}}$ & $39.7 \pm 1.9$ \\
\hline Birth weight (g) & $3475 \pm 520$ & $3425 \pm 651$ & $3194 \pm 536^{b}$ \\
\hline \multicolumn{4}{|l|}{ Image quality (TI) } \\
\hline Poor (but useable) (\%) & 3.5 & - & 8.3 \\
\hline Fair/sufficient (\%) & 36.3 & 47.1 & 38.6 \\
\hline Very good/excellent (\%) & 60.2 & 52.9 & 53.1 \\
\hline
\end{tabular}

$p$-values were obtained using paired $t$-tests for continuous variables and $\chi^{2}$-test for categorical variables. Subjects exposed to prenatal tobacco are matched with nonexposed controls on age and gender.

aSignificantly differs with matched controls with a two-tailed $p<0.05$.

${ }^{b}$ Significantly differs with matched controls with a two-tailed $p<0.01$.

superior temporal, lateral occipital, and precentral cortices. The clusters $\left(>400 \mathrm{~mm}^{2}\right)$ in the right hemisphere were the following: the superior frontal, superior parietal, lateral occipital, precentral, paracentral cortices, and pars opercularis. These analyses survived correction for multiple comparisons (Figure 1). 
Table 2 Prenatal Tobacco Exposure and Global Brain Measures (in $\mathrm{cm}^{3}$ )

\begin{tabular}{|c|c|c|c|c|c|c|}
\hline & \multicolumn{3}{|c|}{$\begin{array}{l}\text { Quit smoking when } \\
\text { pregnancy was known }(N=17)\end{array}$} & \multicolumn{3}{|c|}{$\begin{array}{l}\text { Continued smoking } \\
\text { (multiple trimesters) }(N=96)\end{array}$} \\
\hline & B $(95 \%$ Cl) & p-value & Cohen's d & B $(95 \%$ Cl) & p-value & Cohen's d \\
\hline \multicolumn{7}{|l|}{ Model I } \\
\hline Total brain volume & $60.0(-36.9$ to 156.8$)$ & 0.21 & 0.36 & $-55.3(-93.5$ to -17.0$)$ & $0.005^{\mathrm{a}}$ & -0.37 \\
\hline Cortical volume & $37.2(-24.1$ to 98.5$)$ & 0.22 & 0.36 & $-34.3(-57.9$ to -10.7$)$ & $0.005^{\mathrm{a}}$ & -0.39 \\
\hline Ventricular volume & $-0.5(-6.1$ to 6.1$)$ & 0.98 & -0.01 & $-1.6(-3.0$ to -0.3$)$ & 0.02 & -0.33 \\
\hline \multicolumn{7}{|l|}{ Model II } \\
\hline Total brain volume & I24.4 (I0.3 to 238.4$)$ & 0.04 & & $-62.6(-102.4$ to -22.7$)$ & $0.002^{\mathrm{a}}$ & \\
\hline Cortical volume & 77.6 (3.1 to 152.2$)$ & 0.04 & & $-38.7(-63.4$ to -13.9$)$ & $0.003^{\mathrm{a}}$ & \\
\hline Cortical gray matter volume & 87.5 (9.0 to 166.0) & 0.03 & & $-43.7(-71.4$ to -15.9$)$ & $0.002^{\mathrm{a}}$ & \\
\hline \multicolumn{7}{|l|}{ Model II + birth weight } \\
\hline Total brain volume & $86.7(-60.6$ to 233.9$)$ & 0.22 & & $-59.7(-102.4$ to -17.1$)$ & $0.007^{\mathrm{a}}$ & \\
\hline Cortical volume & 60.7 ( -38.0 to 159.5$)$ & 0.19 & & $-36.5(-62.9$ to -9.9$)$ & $0.008^{\mathrm{a}}$ & \\
\hline Cortical gray matter volume & $63.7(-38.4$ to 165.8$)$ & 0.19 & & $-41.2(-70.9$ to -11.4$)$ & $0.007^{\mathrm{a}}$ & \\
\hline Cortical white matter volume & $30.2(-24.3$ to 84.7$)$ & 0.24 & & $-19.4(-35.6$ to -3.2$)$ & 0.02 & \\
\hline Ventricular volume & $-3.4(-13.4$ to 6.6$)$ & 0.46 & & $-0.9(-2.4$ to 0.7$)$ & 0.29 & \\
\hline
\end{tabular}

Paired multiple linear regression analyses were used. All subjects were matched on age and gender.

Model I is unadjusted. Model II was adjusted for maternal education, ethnicity, and alcohol use during pregnancy.

Bs represent the differences in volumes $\left(\mathrm{cm}^{3}\right)$ between tobacco-exposed and nonexposed children. Negative values represent differences in volumes where the tobacco-exposed children have smaller brain volumes than those who were unexposed. Positive values represent differences in volumes where the tobacco-exposed children have larger brain volumes than those who were unexposed. p-values were obtained using two-tailed tests.

a $p$-value survived familywise error correction (Bonferroni) for multiple testing.

We then extracted the cortices as a region of interest ( $>400 \mathrm{~mm}^{2}$ in both hemispheres) and examined the association of prenatal tobacco exposure and cortical thickness using linear regression analyses that took into account the covariates (Table 4). Continued maternal smoking was significantly associated with thinner cortices of the superior frontal region $(B=-0.08,95 \% \mathrm{CI}:-0.15$ to $-0.0, p=0.02)$, the superior parietal region $(B=-0.06,95 \%$ CI: -0.11 to $-0.02, p=0.007)$, the lateral occipital region $(B=-0.04$, $95 \%$ CI: -0.09 to $-0.01, p=0.04)$, and the precentral region $(B=-0.07,95 \%$ CI: -0.12 to $-0.02, p=0.009)$; these associations remained present after adjusting for the covariates. No associations were observed between early pregnancy tobacco exposure and cortical thickness (Table 3).

No associations between maternal smoking and cortical surface area were observed. No dose-response relationship between prenatal tobacco exposure and brain morphology was observed.

\section{Behavioral and Emotional Problems}

Table 4 shows that continued maternal smoking was associated with increased scores of affective problems $(B=1.00,95 \% \quad C I: 0.31-1.69, \quad p=0.005)$ and anxiety $(B=0.88,95 \%$ CI: $0.09-1.68, p=0.03)$. When adjusting for covariates, the association between continued maternal smoking and affective problems remained present, whereas the relation between continued smoking and anxiety were explained by the covariates (Table 4). Subsequently, we

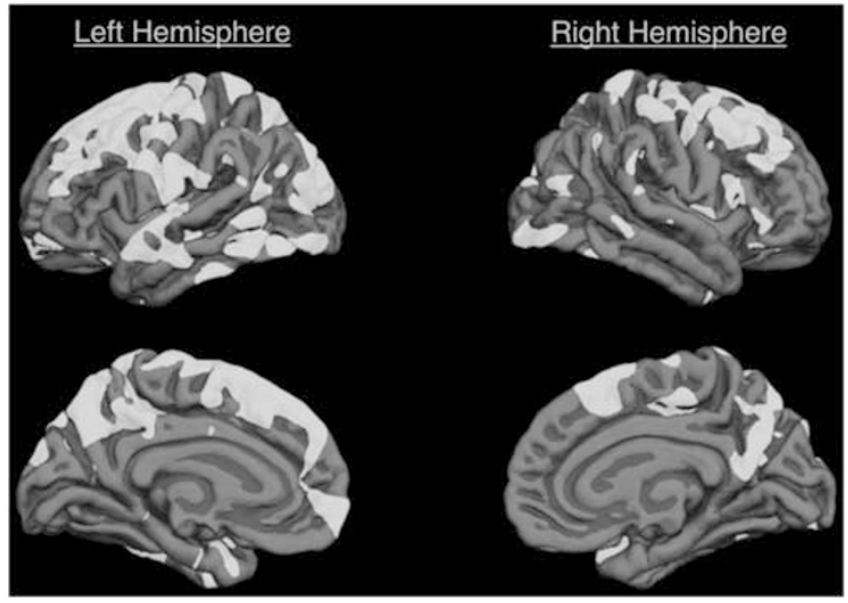

Figure I Differences in cortical thickness between tobacco-exposed (continued maternal smoking) and unexposed children. The colored areas (white in print/orange in web version) on the surface map represent clusters that are thinner in tobacco-exposed (continued maternal smoking) children as compared with unexposed controls in the left and right hemisphere. All clusters in the left hemisphere (including the superior frontal, superior parietal, inferior parietal, precentral, and lateral orbitofrontal cortices) and the clusters in the right hemisphere (including the superior frontal, superior parietal, and precentral cortices) survived the clusterwise (simulation with 5000 iterations) correction for multiple comparisons $(p<0.05)$.

investigated whether the observed differences in brain morphology could explain this association. The association between continued maternal tobacco smoking and affective 
Table 3 Prenatal Tobacco Exposure and Cortical Thickness (in mm)

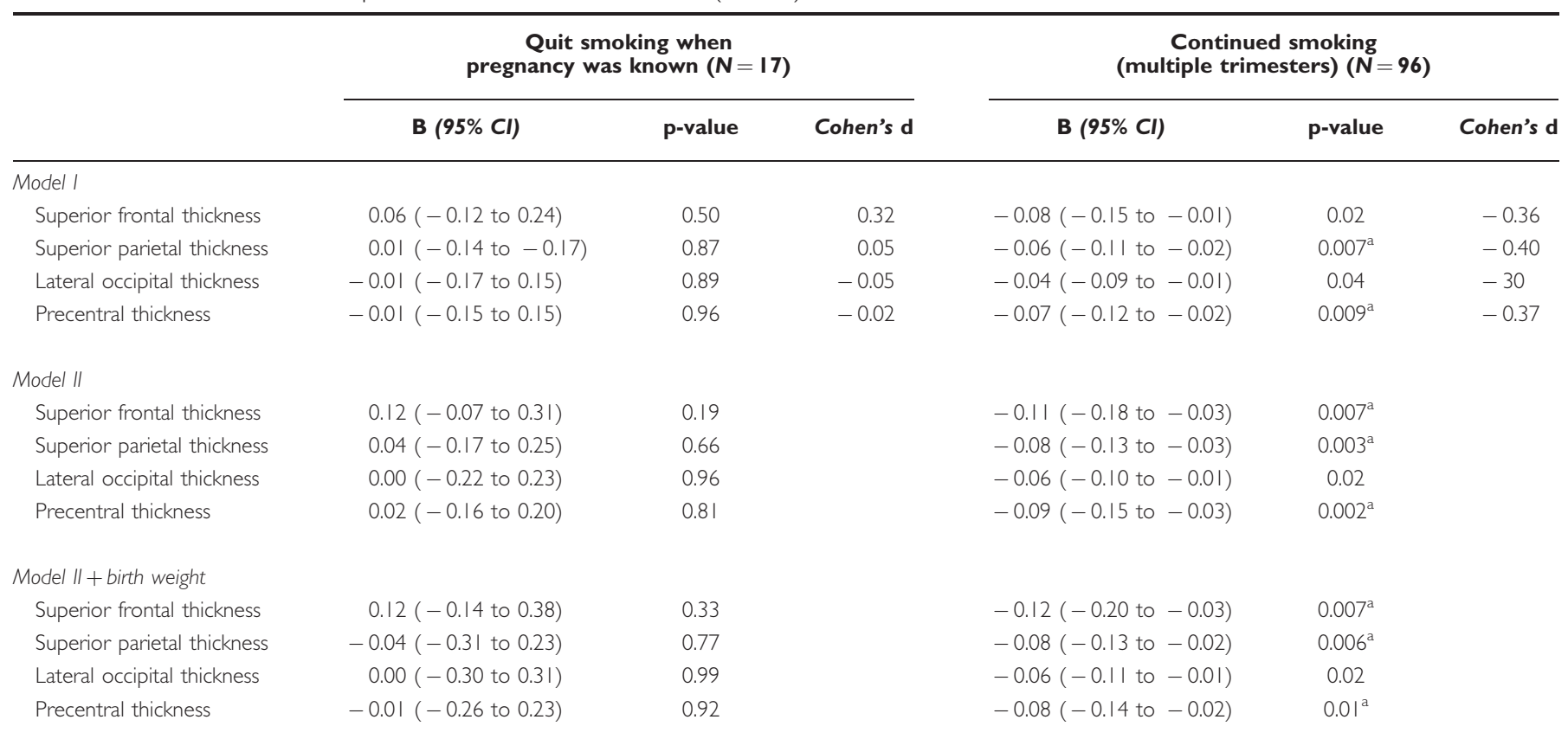

Paired multiple linear regression analyses were used. All subjects were matched on age and gender. Model I is unadjusted. Model II was adjusted for maternal education, ethnicity, and alcohol use during pregnancy.

Bs represent the differences in thickness $(\mathrm{mm})$ between tobacco-exposed and nonexposed children. Negative values represent differences in thickness where the tobacco-exposed children have thinner brain cortices than those who were unexposed. Positive values represent differences in thickness where the tobacco-exposed children have thicker brain cortices than those who were unexposed. $p$-values were obtained using two-tailed tests.

${ }^{a} p$-value survived Bonferroni correction for multiple testing.

problems was not explained by total brain volume, as the association remained present after taking to account total brain volume $(B=1.10,95 \%$ CI: $0.25-1.94, p=0.01)$. We then investigated whether the observed thinner cortices (four clusters) explained the association. The results demonstrated that the relation between continued maternal smoking and affective problems was explained by the thinner superior frontal cortex $(B=0.78 ; 95 \% \mathrm{CI}$ : -0.05 to $1.61, p=0.07)$, thinning of the precentral cortex $(B=0.76$; $95 \%$ CI: -0.05 to $1.57, p=0.07)$, and not explained by the difference in superior parietal thinning $(B=0.91$; $95 \% \mathrm{CI}$ : $0.07-1.76, p=0.04)$ and lateral occipital thinning $(B=0.98$; 95\% CI: $0.15-1.81, p=0.02$ ).

Further, there were direct associations between thickness of the precentral cortex $(B=-2.3 ; 95 \%$ CI: -3.85 to $-0.75, p=0.004)$ and affective problems and thickness of the superior frontal cortex $(B=-1.6 ; 95 \%$ CI: -2.85 to $-0.36, p=0.01)$ and affective problems.

\section{DISCUSSION}

In this prospective study, we examined the association of maternal smoking during pregnancy with brain morphology and behavioral and emotional problems in childhood. We demonstrated that children exposed prenatally to tobacco have smaller brain volumes, smaller cortical gray and white matter volumes, and regional thinning of the superior frontal, superior parietal, lateral occipital, and precentral cortices. Further, children exposed to continued maternal smoking had increased scores on emotional problems, and the observed cortical thinning, in particular in the superior frontal and precentral cortices, explained this association. Moreover, we observed smaller volumes of the caudate and accumbens area in children exposed to continued maternal smoking, but this was explained by the overall smaller total brain volume.

Although this study did not demonstrate a clear doseresponse relation between the quantity of cigarettes smoked during pregnancy and brain morphology or emotional/ behavioral problems, this study clearly demonstrates a duration effect; early pregnancy exposure (quit smoking when knowing about the pregnancy) was not associated with differences in brain morphology nor was it associated with emotional or behavioral problems, whereas continued maternal smoking was. However, differences in the timing of neurodevelopmental processes also could explain these differences.

Largely, our results both support and extend the previous neuroimaging studies in adolescents. For example, prenatal tobacco exposure has been associated with reduction in cortical gray matter and parenchymal volumes in teenagers (Rivkin et al, 2008). Moreover, cortical thickness in the orbitofrontal, middle frontal, and parahippocampal gyrus have been reported to be thinner in adolescents exposed to prenatal tobacco (Toro et al, 2008; Lotfipour et al, 2009). This suggests that the region-specific effects of prenatal tobacco exposure may change over time. In childhood, we observed differences in the parietal and superior frontal regions, however, with ongoing neurodevelopment; these may progress to involve frontal areas in the future. We extended the literature by evaluating potential confounding variables that have not been assessed in other studies. Mothers who smoke during pregnancy may have specific genetic or environmental characteristics that could also 
Table 4 Prenatal Tobacco Exposure and Behavioral and Emotional Problems

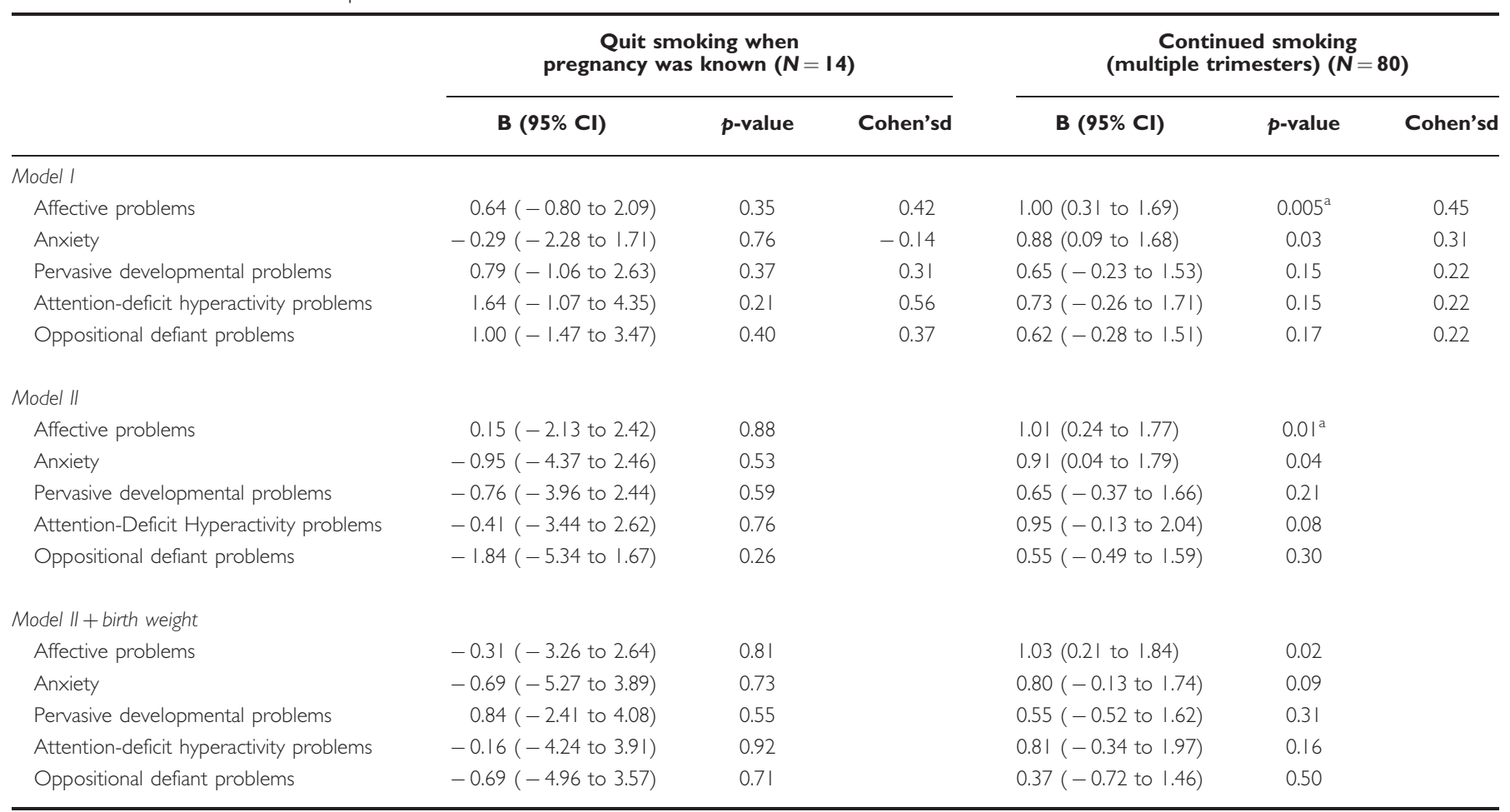

Paired multiple linear regression analyses were used. All subjects were matched on age and gender. Model I is unadjusted. Model II was adjusted for maternal education, ethnicity, and alcohol use during pregnancy.

Bs represent the differences in scores on the Child Behavior Checklist measured at age 6 years between tobacco-exposed and nonexposed children. Negative values represent lower scores (less problems) in the tobacco-exposed children as compared with those who were unexposed. Positive values represent higher scores (more problems) in the tobacco-exposed children as compared with those who were unexposed. p-values were obtained using two-tailed tests.

a $p$-value survived Bonferroni correction for multiple testing.

influence neurodevelopment in their offspring. In our results, the different covariates did not affect the associations between continued maternal smoking and different outcomes. Thus, differences in these brain regions and emotional problems are possibly more likely to be influenced by maternal smoking during fetal neurodevelopment.

A diffusion tensor imaging (DTI) study reported that prenatal and adolescent tobacco exposure affected the regional fractional anisotropy in cortical white matter (Jacobsen et al, 2007). We observed smaller cortical white matter volumes in tobacco-exposed children, but additional studies using DTI will be needed to confirm whether white matter integrity is similarly affected in our subjects. Interestingly, a recent pilot study showed that 12 -year-old children prenatally exposed to tobacco showed greater activation in the inferior parietal regions of the brain during a working memory task (Bennett et al, 2012). This region involves the same cortical area that was found to be thinner in the current study. Additional functional MRI studies in our sample will be valuable to determine whether these structural differences also relate to functional differences in tobacco-exposed children.

Several possible interpretations for our results are plausible. One explanation is that prenatal tobacco exposure directly affects brain development through nicotine, which has been demonstrated in animal studies (Dwyer et al, 2008). For example, a thinner somatosensory cortex in rats has been demonstrated (Roy and Sabherwal, 1994). We have pre- viously demonstrated that prenatal exposure to tobacco was associated with decreased fetal head growth (Roza et al, 2007), and now we demonstrate that these neurodevelopmental effects remain present years after the exposure. Thus, the smaller brain growth in children exposed in utero to smoking does not compensate via neuroplasticity (Huttenlocher, 2002). A potential mechanism for our findings is that prenatal exposure to nicotine affects neuronal migration because we observed that cortical thickness was affected, but cortical surface area was not different in nonexposed controls and tobacco-exposed children. Indeed, early disturbances in neuronal path finding, abnormalities in cell proliferation and differentiation, and disruptions in neurotransmitter systems have been reported in animal studies (Ernst et al, 2001).

Another explanation for our findings is that maternal smoking leads to fetal hypoxia because of vasoconstriction, which leads to reduced blood flow and decreased oxygen to the fetus. This decreased nutrient and oxygen supply might induce long-lasting effects on brain morphology (Abel, 1980).

Finally, the observed effects of continued prenatal tobacco exposure on offspring brain morphology could be epiphenomena of smoking, eg, parental psychopathology, use of other substances, and poor nutrition intake. We minimized the differences between exposed and nonexposed subjects by using a matching procedure; they did not differ in age and gender nor did they differ in other such as maternal ethnicity and education. 
The strengths of this study are the prospective design, including the prospective measures of smoking during pregnancy, the relatively large group that underwent a neuroimaging session, and the young age of the children. In particular, most neuroimaging studies performed examining the association between prenatal tobacco exposure and brain morphology are confounded by smoking in adolescence. In the current study, this was not the case. We also acknowledge that this study has its limitations. We relied on self-reported information for maternal smoking during pregnancy. Using self-reports may have introduced some misclassification because of underreporting of cigarette smoking (Shipton et al, 2009), which may have led to an underestimation of the observed effects. However, in large populations it is sensible to use self-reported information on prenatal tobacco exposure from a cost-effectiveness point of view. Moreover, our study did not include the potential consequences of second-hand smoke after birth, as mothers who smoke during pregnancy are more likely to continue smoking after giving birth and have a partner who also smokes. Unfortunately, data on postnatal parental smoking were missing in a large proportion of our subjects. Therefore, we could not use these data in our analyses. In this study, the group of mothers who stopped smoking when they knew they were pregnant was quite small, and we must be careful interpreting these results. Finally, although this study demonstrates that prenatal exposure to tobacco is associated with thinner cortices and smaller global brain volumes, we must be careful in declaring that this association is causal. There are some studies that suggest a causal relation between maternal smoking and child behavioral problems (Brion et al, 2010). However, there are some studies that suggest that the association between prenatal smoking and problems later in life are not caused by the prenatal exposure, but are due to other familial factors (D'Onofrio et al, 2008; D'Onofrio et al, 2012). Thus, if prenatal tobacco exposure does not have a teratogenic effect, then these familial risk factors should be factors that are involved in brain development.

Overall, our findings suggest long-term effects of prenatal tobacco exposure on brain development and emotional problems in young children. The results of the current study in combination with the existing literature about the long-term effects of prenatal tobacco exposure emphasize the importance of preventing and reducing cigarette smoking during pregnancy. Our findings provide further support for the need of clinical and public health strategies aimed at the prevention of prenatal tobacco exposure of children. More research is needed to explore the structural and functional neurodevelopmental effects of prenatal exposure to tobacco using measures including DTI and functional MRI.

\section{FUNDING AND DISCLOSURE}

The Sophia Children's Hospital Fund (SSWO-553) supported this work financially. The study was made possible by financial support from the Erasmus Medical Center, and the Netherlands Organization for Health Research and Development (Zon MW Geestkracht Program 10.000.1003 and ZonMw TOP 40-00812-98-11021). The funding agencies had no role in the design and conduct of the study, collection, management, analyses and interpretation of the data; and preparation, review or approval of the manuscript and the decision to submit it for publication. Dr Verhulst is head of the Department of Child and Adolescent Psychiatry at Erasmus MC, which publishes the Achenbach System of Empirically Based Assessment (ASEBA) and from which the department receives remuneration. All other authors declare no competing financial interest in relation to the work described.

\section{ACKNOWLEDGEMENTS}

The Generation R Study is conducted by the Erasmus Medical Center in close collaboration with the Municipal Health Service Rotterdam area, the Rotterdam Homecare Foundation, and the Stichting Trombosedienst \& Artsenlaboratorium Rijnmond (STAR), Rotterdam. We gratefully acknowledge the contribution of general practitioners, hospitals, midwives, and pharmacies in Rotterdam.

\section{REFERENCES}

Abbott LC, Winzer-Serhan UH (2012). Smoking during pregnancy: lessons learned from epidemiological studies and experimental studies using animal models. Crit Rev Toxicol 42: 279-303.

Abel EL (1980). Smoking during pregnancy: a review of effects on growth and development of offspring. Hum Biol 52: 593-625.

Bennett DS, Mohamed FB, Carmody DP, Malik M, Faro SH, Lewis M (2012). Prenatal tobacco exposure predicts differential brain function during working memory in early adolescence: a preliminary investigation. Brain Imaging Behav 7: 49-59.

Brion MJ, Victora C, Matijasevich A, Horta B, Anselmi L, Steer C et al (2010). Maternal smoking and child psychological problems: disentangling causal and noncausal effects. Pediatrics 126: e57-e65.

Buka SL, Shenassa ED, Niaura R (2003). Elevated risk of tobacco dependence among offspring of mothers who smoked during pregnancy: a 30-year prospective study. Am J Psychiatry 160: $1978-1984$.

Cnattingius S (2004). The epidemiology of smoking during pregnancy: smoking prevalence, maternal characteristics, and pregnancy outcomes. Nicotine Tob Res 6(Suppl 2): S125-S140.

Cornelius MD, Leech SL, Goldschmidt L, Day NL (2000). Prenatal tobacco exposure: is it a risk factor for early tobacco experimentation? Nicotine Tob Res 2: 45-52.

D’Onofrio BM, Rickert ME, Langstrom N, Donahue KL, Coyne CA, Larsson $\mathrm{H}$ et al (2012). Familial confounding of the association between maternal smoking during pregnancy and offspring substance use and problems. Arch Gen Psychiatry 69: 1140-1150.

D’Onofrio BM, Van Hulle CA, Waldman ID, Rodgers JL, Harden $\mathrm{KP}$, Rathouz PJ et al (2008). Smoking during pregnancy and offspring externalizing problems: an exploration of genetic and environmental confounds. Dev Psychopathol 20: 139-164.

Derauf C, Kekatpure M, Neyzi N, Lester B, Kosofsky B (2009). Neuroimaging of children following prenatal drug exposure. Semin Cell Dev Biol 20: 441-454.

DiFranza JR, Lew RA (1995). Effect of maternal cigarette smoking on pregnancy complications and sudden infant death syndrome. J Fam Pract 40: 385-394.

Ducharme S, Hudziak JJ, Botteron KN, Albaugh MD, Nguyen TV, Karama $S$ et al (2012). Decreased regional cortical thickness and thinning rate are associated with inattention symptoms in healthy children. J Am Acad Child Adolesc Psychiatry 51(18-27): e12.

Dwyer JB, Broide RS, Leslie FM (2008). Nicotine and brain development. . Birth Defects Res C Embryo Today 84: 30-44. 
Ekblad M, Gissler M, Lehtonen L, Korkeila J (2010). Prenatal smoking exposure and the risk of psychiatric morbidity into young adulthood. Arch Gen Psychiatry 67: 841-849.

Ekblad M, Korkeila J, Parkkola R, Lapinleimu H, Haataja L, Lehtonen L (2010). Maternal smoking during pregnancy and regional brain volumes in preterm infants. J Pediatr 156: e181.

Ernst M, Moolchan ET, Robinson ML (2001). Behavioral and neural consequences of prenatal exposure to nicotine. J Am Acad Child Adolesc Psychiatry 40: 630-641.

Fischl B, Dale AM (2000). Measuring the thickness of the human cerebral cortex from magnetic resonance images. Proc Natl Acad Sci USA 97: 11050-11055.

Fischl B, Salat DH, Busa E, Albert M, Dieterich M, Haselgrove C et al (2002). Whole brain segmentation: automated labeling of neuroanatomical structures in the human brain. Neuron 33: 341-355.

Fischl B, Salat DH, van der Kouwe AJ, Makris N, Segonne F, Quinn BT et al (2004). Sequence-independent segmentation of magnetic resonance images. Neuroimage 23(Suppl 1): S69-S84.

Ghosh SS, Kakunoori S, Augustinack J, Nieto-Castanon A, Kovelman I, Gaab N et al (2010). Evaluating the validity of volume-based and surface-based brain image registration for developmental cognitive neuroscience studies in children 4 to 11 years of age. Neuroimage 53: 85-93.

Haghighi A, Schwartz DH, Abrahamowicz M, Leonard GT, Perron M, Richer L et al (2013). Prenatal exposure to maternal cigarette smoking, amygdala volume, and fat intake in adolescence. JAMA Psychiatry 70: 98-105.

Hagler DJ Jr, Saygin AP, Sereno MI (2006). Smoothing and cluster thresholding for cortical surface-based group analysis of fMRI data. Neuroimage 33: 1093-1103.

Han X, Jovicich J, Salat D, van der Kouwe A, Quinn B, Czanner S et al (2006). Reliability of MRI-derived measurements of human cerebral cortical thickness: the effects of field strength, scanner upgrade and manufacturer. Neuroimage 32: 180-194.

Huizink AC, Mulder EJ (2006). Maternal smoking, drinking or cannabis use during pregnancy and neurobehavioral and cognitive functioning in human offspring. Neurosci Biobehav Rev 30: 24-41.

Huttenlocher PR (2002). Neural plasticity: the effects of environment on the development of the cerebral cortex. Harvard University Press: Cambridge, MA, London.

Ivanova MY, Achenbach TM, Rescorla LA, Harder VS, Ang RP, Bilenberg $\mathrm{N}$ et al (2010). Preschool psychopathology reported by parents in 23 societies: testing the seven-syndrome model of the child behavior checklist for ages 1.5-5. J Am Acad Child Adolesc Psychiatry 49: 1215-1224.

Jacobsen LK, Picciotto MR, Heath CJ, Frost SJ, Tsou KA, Dwan RA et al (2007). Prenatal and adolescent exposure to tobacco smoke modulates the development of white matter microstructure. J Neurosci 27: 13491-13498.

Jaddoe VW, van Duijn CM, van der Heijden AJ, Mackenbach JP, Moll HA, Steegers EA et al (2010). The Generation R Study: design and cohort update 2010. Eur J Epidemiol 25: 823-841.

Kuperberg GR, Broome MR, McGuire PK, David AS, Eddy M, Ozawa F et al (2003). Regionally localized thinning of the cerebral cortex in schizophrenia. Arch Gen Psychiatry 60: 878-888.

Liu J, Lester BM, Neyzi N, Sheinkopf SJ, Gracia L, Kekatpure M et al (2013). Regional brain morphometry and impulsivity in adolescents following prenatal exposure to cocaine and tobacco. JAMA Pediatr 167: 348-354.

Lotfipour S, Ferguson E, Leonard G, Perron M, Pike B, Richer L et al (2009). Orbitofrontal cortex and drug use during adolescence: role of prenatal exposure to maternal smoking and BDNF genotype. Arch Gen Psychiatry 66: 1244-1252.

Nielsen A, Hannibal CG, Lindekilde BE, Tolstrup J, Frederiksen K, Munk C et al (2006). Maternal smoking predicts the risk of spontaneous abortion. Acta Obstet Gynecol Scand 85: 1057-1065.

O’Donnell S, Noseworthy MD, Levine B, Dennis M (2005). Cortical thickness of the frontopolar area in typically developing children and adolescents. Neuroimage 24: 948-954.

Paus T, Nawazkhan I, Leonard G, Perron M, Pike GB, Pitiot A et al (2008). Corpus callosum in adolescent offspring exposed prenatally to maternal cigarette smoking. Neuroimage 40: 435-441.

Reuter M, Schmansky NJ, Rosas HD, Fischl B (2012). Withinsubject template estimation for unbiased longitudinal image analysis. Neuroimage 61: 1402-1418.

Rivkin MJ, Davis PE, Lemaster JL, Cabral HJ, Warfield SK, Mulkern RV et al (2008). Volumetric MRI study of brain in children with intrauterine exposure to cocaine, alcohol, tobacco, and marijuana. Pediatrics 121: 741-750.

Rogers JM (2009). Tobacco and pregnancy. Reprod Toxicol 28: $152-160$.

Rosas HD, Liu AK, Hersch S, Glessner M, Ferrante RJ, Salat DH et al (2002). Regional and progressive thinning of the cortical ribbon in Huntington's disease. Neurology 58: 695-701.

Roy TS, Andrews JE, Seidler FJ, Slotkin TA (1998). Nicotine evokes cell death in embryonic rat brain during neurulation. J Pharmacol Exp Ther 287: 1136-1144.

Roy TS, Sabherwal U (1994). Effects of prenatal nicotine exposure on the morphogenesis of somatosensory cortex. Neurotoxicol Teratol 16: 411-421.

Roza SJ, Verburg BO, Jaddoe VW, Hofman A, Mackenbach JP, Steegers EA et al (2007). Effects of maternal smoking in pregnancy on prenatal brain development. The Generation $\mathrm{R}$ Study. Eur J Neurosci 25: 611-617.

Salat DH, Buckner RL, Snyder AZ, Greve DN, Desikan RS, Busa E et al (2004). Thinning of the cerebral cortex in aging. Cereb Cortex 14: 721-730.

Shipton D, Tappin DM, Vadiveloo T, Crossley JA, Aitken DA, Chalmers J (2009). Reliability of self reported smoking status by pregnant women for estimating smoking prevalence: a retrospective, cross sectional study. BMJ 339: b4347.

Slotkin TA (2004). Cholinergic systems in brain development and disruption by neurotoxicants: nicotine, environmental tobacco smoke, organophosphates. Toxicol Appl Pharmacol 198: $132-151$.

Steliga MA, Dresler CM (2011). Epidemiology of lung cancer: smoking, secondhand smoke, and genetics. Surg Oncol Clin N Am 20: 605-618.

Tellegen PJ, Winkel M, Wijnberg-Williams B, Laros JA (2005). SON-R 2,5-7: Snijders-Oomen niet-verbale intelligentietest: Verantwoording en handleiding. Hogrefe Uitgevers: Amsterdam, The Netherlands.

Toro R, Leonard G, Lerner JV, Lerner RM, Perron M, Pike GB et al (2008). Prenatal exposure to maternal cigarette smoking and the adolescent cerebral cortex. Neuropsychopharmacology 33: 1019-1027.

Weissman MM, Warner V, Wickramaratne PJ, Kandel DB (1999). Maternal smoking during pregnancy and psychopathology in offspring followed to adulthood. $\mathrm{J} \mathrm{Am}$ Acad Child Adolesc Psychiatry 38: 892-899.

White T, El Marroun H, Nijs I, Schmidt M, van der Lugt A, Wielopolki PA et al (2013). Pediatric population-based neuroimaging and the Generation $\mathrm{R}$ Study: the intersection of developmental neuroscience and epidemiology. Eur J Epidemiol 28: $99-111$. 